\title{
INVESTIGAÇÃO DA MEMÓRIA EM CRIANÇAS EM IDADES ESCOLAR E PRÉ-ESCOLAR ${ }^{1}$
}

\author{
Carmem Beatriz Neufeld \\ Priscila Goergen Brust ${ }^{\#}$ \\ Marcelle Thays Gomes da Silva"
}

\begin{abstract}
RESUMO. O presente trabalho visou investigar experimentalmente, a susceptibilidade das memórias de crianças à distorção, através do estudo das falsas memórias, que são lembranças de eventos que, na realidade, não ocorreram. O desempenho da memória foi avaliado em 57 crianças em idade escolar e pré-escolar, por meio da apresentação de sequências de frases simples que representam informações do cotidiano das crianças. Os resultados indicaram que a memória não foi imune a erros e distorções, ou seja, as crianças se lembraram falsamente de informações a respeito do material estudado. Uma comparação entre as faixas etárias permitiu identificar que as crianças em idade escolar produziram menos falsas memórias que aquelas em idade pré-escolar. Assim, a escolaridade das criança parece ter auxiliado as crianças mais velhas na identificação das informações verdadeiras e na rejeição de falsas memórias.
\end{abstract}

Palavras-chave: Memória; falsas memórias; crianças.

\section{INVESTIGATION OF MEMORY IN CHILDREN OF SCHOOL AND PRESCHOOL AGE}

\begin{abstract}
The current essay aimed to investigate the susceptibility of children to memory distortion by assessing false memories, which are the remembrance of events which actually did not occur. Memory performance was evaluated in 57 school and preschool children through the presentation of a series of simple sentences that represent children's daily life. Results suggest that memory has not been immune from errors and distortions, that is, children falsely remembered information about the studied material. A comparison between the age groups identified that the school children produced fewer false memories than those in preschool. Thus, schooling of children seemed to help older children in identifying correct information and rejecting the false ones.
\end{abstract}

Key words: Memory; false memories; children.

\section{INVESTIGACIÓN DE MEMORIA EN NIÑOS DE EDAD ESCOLAR Y PREESCOLAR}

RESUMEN. El presente trabajo tuvo como objetivo investigar experimentalmente la suceptibilidad de la distorsión de la memoria en los niños através del estudio de las falsas memorias, que son los recuerdos de eventos que no ocurrieron en realidad. El desempeño de la memoria fue evaluado en 57 niños en edad escolar y preescolar, por medio de la presentación de secuencias de frases sencillas que representan informaciones cotidianas en niños. Los resultados indicaron que la memoria no fue inmune a errores y distorsiones, es decir, los niños recordaron falsamente informaciones sobre el material estudiado. Una comparación entre los grupos de edades permitió identificar que los niños en edad escolar producían menos falsas memorias en comparación con los de edad preescolar. La escolaridad de los niños por lo tanto parece ayudar a los niños mayores en la identificación de las informaciones verdaderas, así como en el rechazo de falsas memorias.

Palabras-clave: Memoria; falsas memorias; niños.

\footnotetext{
Apoio: Fundação Araucária.

Doutora em Psicologia pela Pontifícia Universidade Católica do Rio Grande do Sul. Professora do Departamento de Psicologia da Faculdade de Filosofia, Ciências e Letras de Ribeirão Preto da Universidade de São Paulo, Brasil.

\# Mestre em Psicologia pela Pontifícia Universidade Católica do Rio Grande do Sul, Brasil. Mestranda em Human Developent pela Cornell University, Estados Unidos.

II Psicóloga pela Faculdade Assis Gurgacz, formação em Terapia Cognitiva pela OsteoFísio Terapias, Brasil.
} 
O fenômeno de recordar-se de algo que não ocorreu ou que na realidade não foi vivenciado - as falsas memórias - vem sendo uma área de grande interesse dos pesquisadores, devido, principalmente, a suas implicações legais relacionadas à habilidade de crianças em relatar fidedignamente fatos vivenciados, tanto como vítimas de abusos quanto como testemunhas oculares de contravenções em geral. Alguns pesquisadores têm destacado os riscos de algumas técnicas de entrevista forense na qualidade e na confiabilidade das declarações de crianças (Brainerd, Reyna, \& Ceci, 2008; Henry \& Ggudjonsson, 2004; Pisa \& Stein, 2006).

Uma das teorias que melhor explicam as falsas memórias é a Teoria do Traço Difuso (do inglês, Fuzzy Trace Theory), Brainerd e Reyna, 2005; Reyna e Brainerd, (1995), segundo a qual a memória é concebida como dois sistemas independentes, e não unitários de memória: a memória literal e a memória de essência. Esses dois sistemas de memória diferem quanto ao conteúdo de suas representações. A memória literal representa os detalhes superficiais e específicos da experiência, codificados episodicamente, enquanto a memória da essência representa o sentido, os padrões e o significado da experiência vivenciada.

Outra premissa da Teoria do Traço Difuso é que representações literais e da essência da mesma experiência são codificadas em paralelo e armazenadas em separado, ou seja, não há sobreposição implementacional absoluta dos correlatos neurais das representações do sentido de uma experiência (memória de essência) às suas representações episódicas. Essa dissociação dos dois sistemas de memória torna os traços literais mais próximos de uma recuperação verdadeira da informação do que os traços de essência (Brainerd \& Reyna, 2005).

Não obstante, ainda não foi alcançada a elucidação de como ocorre a produção de falsas memórias em crianças, uma vez que na literatura são encontrados resultados incongruentes (Brainerd \& Mojardin 1998; Howe, 2005). Faz necessário compreender o fenômeno das falsas memórias em todas as faixas etárias, pois ele apresenta implicações práticas em áreas clínicas e jurídicas, por exemplo, tanto por meio de sucessivos questionamentos sobre um evento quanto pela maneira como tais questionamentos são conduzidos (Welter \& Feix, 2010).

Em uma revisão recente, Brainerd et al. (2008) ressaltaram que as falsas memórias diminuem com a passagem da infância para a adolescência. Por outro lado, com o aumento da idade também aumenta a acurácia da memória. Essa contradição entres as afirmações dos autores deve-se aos diferentes tipos de evocação de falsas memórias: confabulação e associação semântica. O primeiro é mais primitivo e aponta para uma menor maturidade mental, enquanto o segundo reflete a eficácia das rotas de associação semântica elaboradas. Além disso, em comparação com os adultos, as crianças produzem mais falsas memórias, principalmente quando é longo o intervalo entre a fase de estudo e a de teste. Segundo os autores, essa diferença deve-se à maior facilidade dos adultos para distinguir entre uma lembrança verdadeira e uma falsa. Essa facilidade parece estar relacionada à memória da essência, já que: (a) traços da essência de material com sentido deveriam ficar mais fortes com a idade, e (b) traços da essência são a base dos alarmes falsos, e não de rejeições corretas.

Um estudo de Howe (2005) confirma esse padrão mnemônico, mesmo entre crianças de diferentes faixas etárias, em relação a listas de palavras semanticamente associadas. O autor comparou o desempenho da memória de crianças em dois momentos do período escolar (7 e 11 anos) com o de crianças em idade préescolar (5 anos). Segundo o autor, as crianças mais velhas (11 anos) recordaram mais informações verdadeiras e falsas do que as de 7 e 5 anos; já as crianças de 7 anos recordaram mais informações verdadeiras do que as de 5 anos, não havendo diferença entre as falsas recordações. Na tentativa de compreender esse achado, alguns autores sugerem que a capacidade de extrair as informações de essência de informações semanticamente associadas, culminando em um aumento das falsas lembranças, está associada ao aumento da idade já nas crianças (Brainerd, Forrest, Karibian, \& Reyna, 2006; Lampinen, Leding, Reed, \& Odegard, 2006). Um estudo semelhante realizado por Brainerd e Reyna (2007) entre crianças com idades médias de 6,10 e 14 anos enfatiza que ocorre um aumento das falsas lembranças com o aumento da idade quando as palavras estavam semanticamente associadas.

Quando se trata de estudar a dissociação da informação recuperada por crianças, por outro lado, Brainerd e Mojardin (1998) investigaram o desempenho da memória em crianças de 6, 8 e 11 anos usando frases curtas sobre informações do cotidiano. Os resultados sugerem que as falsas memórias são mais estáveis com o passar do tempo, independentemente da faixa etária. Esse estudo levanta considerações importantes sobre o desempenho da memória em crianças estar associado à passagem do tempo. Esse achado foi denominado efeito de persistência das falsas memórias, por serem estas mais duradouras e mais consistentes do que as lembranças relativas a experiências reais.

Os estudos de Seamon, Luo, Schlegel, Greene, e Goldenberg (2000) sustentam essa hipótese de que as falsas 
memórias são estáveis independentemente da faixa etária quando testadas dias após a fase de estudo utilizando-se listas de palavras semanticamente associadas. Nesse estudo, a memória de crianças (média etária de 6 e 11 anos) e adultos (universitários) foi avaliada imediatamente após a fase de estudo ou três dias depois. Os resultados indicaram uma estabilidade das falsas memórias no teste posterior (três dias depois) tanto em adultos como em crianças. Esse achado para o teste posterior foi acompanhado da diminuição da recuperação de informações verdadeiras e do aumento das falsas memórias em comparação ao teste imediato. Uma possível explicação para as diferenças na durabilidade da memória é que as respostas, quando têm uma base mnemônica, estão sustentadas, principalmente, por representações literais instáveis, em função da suscetibilidade a efeitos de interferência, tornando-se mais rapidamente inacessíveis, enquanto lembranças falsas estão baseadas, fundamentalmente, em representações da essência da experiência, as quais são bem mais estáveis (Neufeld, Brust, \& Stein, 2010).

Tais resultados, apesar de incongruentes entre si, também vão ao encontro dos de estudos anteriores sobre falsas memórias em crianças, os quais sugerem que a acurácia da memória se desenvolve com o aumento da idade, porém a suscetibilidade à sugestão de falsa informação é maior em crianças pré-escolares (Bruck, Ceci, \& Hembrooke, 2002). Este fato parece estar relacionado à suscetibilidade das crianças a distorcer uma informação com base na memória que já possuem sobre um evento. Uma alternativa para esta explicação foi proposta por Brainerd e Reyna (1998), os quais sugerem que, com o aumento da idade, a criança desenvolve uma habilidade de reter os traços literais armazenados sobre as informações verdadeiras; a recuperação de tais traços dá suporte à correta rejeição de informações falsas quando são apresentadas em um teste de memória.

Os resultados dos estudos destacados até o presente momento enfatizam a incongruência de resultados observados na literatura. Por outro lado, cabe destacar que existem diferenças entre os vários estudos no que tange à comparação entre diversas faixas etárias (crianças pré-escolares, escolares em mais de uma faixa etária e pessoas adultas), à utilização de materiais diferentes (listas de palavras e frases complexas) e à testagem da memória em diferentes momentos (o imediato e o posterior). Tais contradições foram destacadas em uma revisão feita por Barbosa, Ávila, Feix e Grassi-Oliveira (2010), sugerindo uma escassez de estudos padronizados na literatura e instigando o desenvolvimento de mais pesquisas na área.

$\mathrm{O}$ presente trabalho buscou investigar experimentalmente alguns dos mecanismos responsáveis pelas falsas memórias em crianças em idade pré-escolar em comparação a crianças em idade escolar em um teste de memória imediato, visando a suprir algumas das divergências metodológicas apontadas na literatura em relação à falsificação da memória em crianças. Para tanto, aplicou-se um instrumento que possibilitasse o estudo da produção de falsas memórias em crianças por meio da apresentação de frases estruturadas, semelhantemente ao material utilizado por Brainerd e Mojardin (1998).

Diferentemente dos estudos destacados até o presente momento, as faixas etárias foram definidas de maneira mais ampla (crianças pré-escolares de 4 a 6 anos e crianças em idade escolar de 8 a 10 anos), no intuito de possibilitar a observação do desempenho das crianças conforme seu grau de escolaridade, e não apenas conforme sua idade. Para que essa investigação não contradissesse as diferenças de escolaridade, o presente estudo não testou crianças com sete anos, porque estariam no início da idade escolar dentro da norma brasileira até o momento da coleta de dados. As crianças acima de 8 anos estariam iniciando o desenvolvimento da capacidade de extração da essência do conteúdo da frase e estariam mais aptas a distinguir entre uma memória verdadeira e uma falsa memória.

$\mathrm{O}$ instrumento utilizado foi adaptado para a língua portuguesa no Brasil por Neufeld e Stein (2003), da versão em inglês de Stein (1998), e constitui-se de estruturas frasais complexas - como, por exemplo, sentenças -, visando a atingir crianças em idade préescolar de maneira semelhante à usada para atingir crianças em idade escolar, ou seja, com o auxílio do contexto na extração de traços de essência. Nesse sentido, espera-se que crianças em idade pré-escolar apresentem mais falsas memórias do que crianças em idade escolar, em função da habilidade destas em recuperar tanto traços literais como traços de essência que auxiliam na rejeição de informações falsas.

\section{MÉTODO}

\section{Delineamento}

$\mathrm{O}$ presente estudo utilizou um delineamento fatorial misto, com duas variáveis de estudo. A primeira variável, denominada grupo, referia-se à faixa etária escolar das crianças, que poderiam pertencer ao grupo escolar ou pré-escolar. A segunda variável, denominada item, dizia respeito aos itens do teste de memória, que poderiam ser alvo, distrator relacionado ou distrator não relacionado. A variável dependente foi o desempenho da memória no teste de reconhecimento. 


\section{Participantes}

Participaram do estudo 57 crianças de uma escola particular do Interior do Estado do Paraná. As crianças foram divididas em dois grupos, conforme a faixa etária em que se encontravam: escolar e pré-escolar. As 27 crianças em idade escolar tinham de 8 a 10 anos $(\mathrm{M}=9, \mathrm{DP}=0,78)$, e as 30 pré-escolares, entre 4 e 6 $\operatorname{anos}(\mathrm{M}=5,43, \mathrm{DP}=0,57)$.

\section{Instrumentos e procedimentos}

Após o contato com a escola, para a qual foi encaminhada uma carta explicando a natureza do estudo, seus objetivos e procedimentos, os pais de todas as crianças incluídas na faixa etária do estudo foram informados sobre a realização da pesquisa e convidados a participar. A amostra foi composta por todas as crianças cujos pais ou responsáveis aceitaram o convite mediante a assinatura de um termo de consentimento livre e esclarecido.

A coleta de dados foi realizada, individualmente e em um único encontro por uma experimentadora do sexo feminino. A testagem ocorreu em uma sala silenciosa na própria escola da criança. Após um período inicial de familiarização, a criança foi solicitada a prestar atenção a uma lista de frases que iria ouvir e da qual posteriormente deveria lembrar-se. Quando percebia que a criança não havia entendido, a experimentadora repetia as instruções até ter certeza da completa compreensão da criança de qual era o seu papel. Foram utilizados oito itens de prática, no intuito de familiarizar a criança com os procedimentos do teste de memória.

Os instrumentos utilizados foram os produzidos por Stein (1998) e adaptados para a língua portuguesa por Neufeld e Stein (2003) para testagem de falsas memórias em crianças. O procedimento experimental consistia de um material-alvo, uma atividade de distração, uma atividade de sugestão de falsa informação e um teste de memória de múltipla escolha. No presente estudo apenas o material de sugestão não foi utilizado, pelo fato de não ser objetivo desse estudo verificar o efeito da sugestão sobre a memória das crianças.

O material-alvo utilizado para avaliar o desempenho da memória das crianças foi uma sequência de frases simples que representam informações do cotidiano da criança, como "Ele recebeu visitas no seu aniversário". O material consistia em 36 frases audiogravadas, com intervalo de três segundos entre cada uma delas, apresentadas de forma aleatória, exceto pelo controle no sentido de que quatro frases de isolamento fossem apresentadas no início da lista e quatro, no final. As frases de isolamento foram inseridas com o intuito de controlar os efeitos de primazia e recência postulado por Ebbinhaus (Murdock, 1962), ou seja, a maior facilidade de recordação das primeiras e últimas frases de uma lista. Além disso, as frases de isolamento serviram como itens de prática do teste de memória de múltipla escolha.

Em seguida as crianças realizaram uma atividade de distração, proposta com o objetivo de desviar a atenção das frases originais. Essa atividade consistia em uma tarefa não verbal de procurar personagens entre a multidão do tipo "Onde está Wally?". Durante um intervalo de sete a dez minutos, as crianças deveriam identificar alguns dos personagens indicados entre inúmeros desenhos de objetos e pessoas em um livro de figuras.

Por fim, foi aplicado um teste de memória de reconhecimento de múltipla escolha. $\mathrm{O}$ teste consistia em uma lista de frases incompletas, como "Ele recebeu no seu aniversário". As frases incompletas eram idênticas às do material original estudado, exceto num aspecto: estava faltando a palavra-chave de cada frase. Quatro palavras eram apresentadas como opções possíveis para completar cada frase. A criança era instruída a selecionar a palavra exata que completava a frase que ela havia escutado anteriormente. A instrução ainda enfatizava que algumas opções poderiam conter palavras semelhantes às originais, porém ela devia escolher a opção que fosse exatamente igual à palavra que completava a frase na lista audiogravada apresentada no início da sessão experimental. Uma fase de treino foi realizada antes do início do teste, assegurando que a criança havia entendido a instrução, utilizando as oito frases de isolamento apresentadas no início e no final da lista original.

O teste de memória de reconhecimento de múltipla escolha consistia de 24 frases acompanhadas de quatro opções de resposta. As opções de resposta poderiam ser alvo, como a palavra "visitas" para o exemplo anterior, ou o distrator relacionado, como a palavra "convidados". Outras duas opções foram acrescentadas no intuito de controlar as respostas de viés da criança, ou seja, o chute, os distratores não relacionados. Esses itens eram informações não semanticamente associadas ao item alvo ou distrator relacionado, embora semanticamente relacionados entre si, como "beijos" e "carinho". A ordem de apresentação das quatro opções de resposta variou aleatoriamente ao longo das frases dos testes de memória.

\section{Análise de dados}

Os resultados foram analisados com base nas três possibilidades de resposta do teste de memória: alvo, 
distrator relacionado ou distrator não relacionado. A criança escolher um item alvo no teste de memória representava uma resposta correta e referia-se a uma medida de memórias verdadeiras (MV). Quando a criança escolhia um item que pertencia à mesma categoria semântica do item alvo, isto é, um distrator relacionado, essa resposta referia-se a uma medida de falsas memórias (FM); já a escolha de um dos dois distratores não relacionados era considerada uma resposta de viés ou chute $(\mathrm{RV})$.

Os dados foram analisados com o auxílio do programa Statistical Package for Social Sciences (SPSS) versão 12.0. Todos os tratamentos estatísticos utilizaram um $\alpha=0,05$ para o teste de hipóteses. Foram realizadas análises post hoc e comparações pareadas com a correção de Bonferroni.

\section{RESULTADOS E DISCUSSÃO}

A partir de uma análise de variância (ANOVA) para grupo versus item do teste de memória, foi observado um efeito principal de item $[\mathrm{F}(2,110)=$ 137,29, p $<0,001]$. Esse resultado foi qualificado por uma análise post hoc, que sugere que os índices de reconhecimento das $\mathrm{MVs}(\mathrm{M}=0,64 ; \mathrm{DP}=0,21)$ foram superiores aos índices das FMs $(\mathrm{M}=0,15$; DP $=0,09)$ e das RV $(\mathrm{M}=0,21 ; \mathrm{DP}=0,14 ; \mathrm{ps}<0,001)$. Este achado corrobora o estudo de Seamon et al.. (2000), o qual enfatiza que uma testagem imediata da memória favorece a recuperação de traços literais, que representam informações específicas do material a ser memorizado. Por outro lado, a produção de FMs foi inferior à de RVs ( $\mathrm{p}<0,01)$, o que sugere um alto nível de chute nas respostas das crianças. Este resultado apresenta evidências semelhantes ao do estudo de Brainerd e Mojardin (1998), que observaram uma produção superior de RVs em comparação à MV em crianças.

Os resultados foram também qualificados pela interação entre grupo e item do teste $[\mathrm{F}(2,110)=$ $11,32, \mathrm{p}<0,001]$. Os dados indicaram que crianças escolares obtiveram índices de $\mathrm{MV}(\mathrm{M}=0,73$; $\mathrm{DP}=$ $0,15)$ superiores aos de FM $(\mathrm{M}=0,12 ; \mathrm{DP}=0,08) \mathrm{e}$ RV $(\mathrm{M}=0,16 ; \quad \mathrm{DP}=0,1 ;$ ps $<0,001)$. Quando observados os resultados obtidos pelas crianças em idade pré-escolar observa-se também uma preponderância das MVs $(\mathrm{M}=0,55$; $\mathrm{DP}=0,22)$ sobre as FMs $(M=0,19$; DP $=0,1)$ e as $\mathrm{RV}(\mathrm{M}=0,26$; DP $=0,15 ; \mathrm{ps}<0,001)$. A memória das crianças em idade pré-escolar demonstrou uma propensão ao chute, uma vez que apenas entre elas observaram-se índices de FM inferiores aos de RV ( $\mathrm{p}<0,001)$. Este resultado apresenta evidências semelhantes às do estudo de Brainerd, Reyna, e Forrest (2002), o qual sugere que crianças mais novas têm maior dificuldade em compreender a essência de informações a serem memorizadas. Como as informações foram armazenadas em duas memórias independentes, a literal e a de essência, que são processadas em paralelo, sua recuperação também é independente, segundo a Teoria do Traço Difuso (Brainerd \& Reyna, 2005).

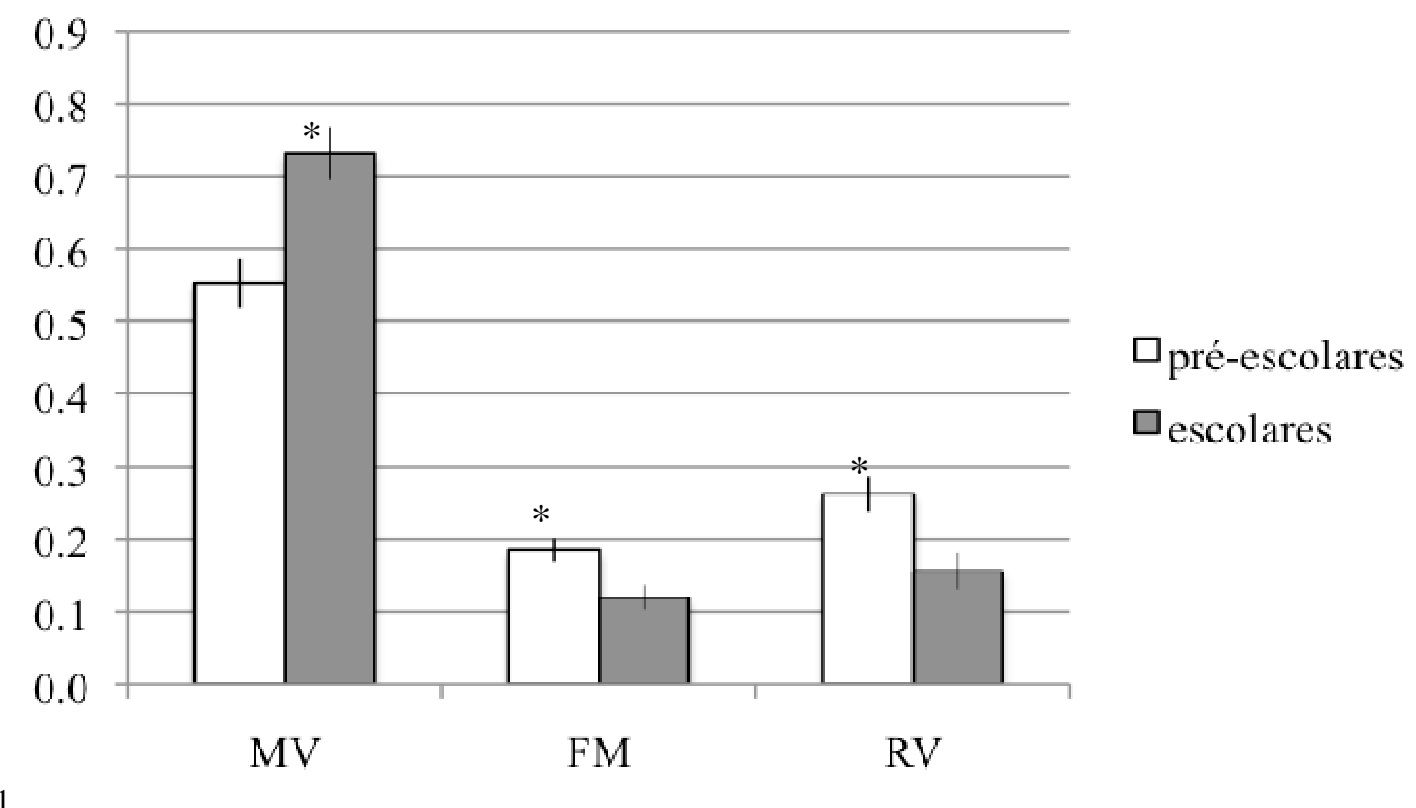

$* \mathrm{p}<0,001$

Figura 1 - Desempenho da memória no teste de reconhecimento entre crianças em idade escolar e pré-escolar. 
A interação entre grupo e item do teste também permite uma comparação entre grupos, indicando que as crianças em idade escolar obtiveram índices de MV superiores aos das pré-escolares $(\mathrm{p}<0,01)$. Dados parecidos foram obtidos em uma amostra de préescolares brasileiros de Neufeld e Stein (2003), e parecem condizer com a hipótese de Brainerd e Reyna (1998), os quais sugerem que crianças em idade escolar desenvolvem a habilidade de reter os traços literais, recuperando-os mais facilmente do que as crianças pré-escolares. Segundo a Teoria do Traço Difuso, a recuperação de traços literais dá suporte à recuperação de informações verdadeiras, mas também sustenta a correta rejeição de informações falsas. Como consequência disso, observa-se também uma diminuição da produção de FMs e de RVs para as crianças em idade escolar em comparação às crianças em idade pré-escolar (ps < 0,01).

Esse resultado, apesar de condizente com a revisão de Brainerd et al. (2008), segundo a qual as falsas memórias diminuiriam com o aumento da idade, é contrário ao postulado de Brainerd e Reyna (2007), que sugere um aumento das falsas memórias com o aumento da idade. Por outro lado, é preciso levar em conta que Brainerd e Reyna (2007) não avaliaram crianças pré-escolares, o que parece indicar que o início dos estudos formais (escolares) favorece a formação de traços de essência. Considerando-se estes aspectos e os estudos com adultos (Sugrue \& Hayne, 2006), parece realmente haver um aumento nos reconhecimentos falsos com a idade, pois, à medida que crescem, os sujeitos se tornam mais hábeis em extrair a essência dos eventos. Esse aumento nas distorções parece vir acompanhado da diminuição no viés, podendo-se levantar a hipótese de que, uma vez mantida a essência da informação, os participantes passam a recorrer menos aos chutes como alternativa de resposta.

Assim, o presente estudo sugere que o aumento das distorções relatado por Brainerd e Reyna (2007) talvez não ocorra na comparação entre préescolares e escolares e sim, na comparação entre pré-adolescentes e adolescentes. Essa hipótese decorre de considerações sobre os dados aqui apresentados, uma vez que a produção de falsas memórias por crianças em idade escolar apresenta valores muito baixos. Apesar disso, por outro lado, a acurácia da memória parece aumentar com o início da educação formal, uma vez que os escolares apresentaram mais memórias verdadeiras, menos falsas memórias e menos respostas de viés do que as crianças pré-escolares.

\section{CONSIDERAÇÕES FINAIS}

O paradigma metodológico empregado na presente pesquisa destaca que o desempenho da memória é melhor para crianças em idade escolar (oito a dez anos). A explicação para estes dados é que, quando respostas corretas têm uma base mnemônica, elas estão sustentadas principalmente por representações literais (Neufeld et al., 2010). As representações literais parecem auxiliar tanto na recuperação de informações-alvo com caráter complexo, como as sentenças apresentadas na fase de estudo e a correta rejeição de distratores, diminuindo a produção de falsas memórias em comparação a crianças em idade pré-escolar (quatro a seis anos). Segundo Brainerd et al. (2008), crianças em idade escolar já apresentam a habilidade de distinguir entre informações verdadeiras e falsas como os adultos, em função do desenvolvimento de traços literais e de essência.

Embora o presente estudo pareça sustentar que a habilidade de reter os traços literais armazenados sobre os alvos e de recordá-los quando o distrator é apresentado no teste de memória se desenvolve com a idade, ainda há uma lacuna na literatura quando tenta explicar o que acontece com as crianças pré-escolares. As crianças pré-escolares, mesmo tendo recuperado menos MVs do que as escolares, parecem ter-se excedido na produção de RVs (que não tem relação semântica com o material alvo) em comparação com a produção de FMs (que está semanticamente relacionada ao material-alvo). Se o material-alvo constituído por frases complexas envoltas em um contexto não favorece a recuperação de lembranças verdadeiras, mas induz a uma recuperação de informações não relacionadas (RV) superior à de informações semanticamente relacionadas (FM), então o tipo de material utilizado não deveria produzir impacto na falsificação da memória em crianças.

Algumas limitações metodológicas podem ter influenciado os resultados do presente estudo, como a apresentação de informações complexas, que pode ter interferido na memória para as informações do material-alvo. Sendo assim, sugere-se que em estudos futuros na busca por compreender o desenvolvimento da memória explorem uma relação mais avançada entre os fatores avaliados no presente estudo e outros possíveis intervenientes, como uma testagem posterior da memória e a apresentação de informações não complexas ou não semanticamente relacionadas. Outros fatores importantes a serem considerados que podem distorcer a memória para a informação original em pesquisas com crianças, especialmente no que 
concerne a diferenças em escolaridade - como, por exemplo, a sugestão de falsas informações -, são destacados por Powell, Jones, e Campbell (2003).

Por fim, a compreensão do processo de distorção mnemônica ao longo do ciclo vital ainda não está clara. Os resultados do presente estudo também parecem apontar a necessidade de estudos que busquem um mapeamento longitudinal nas diferentes fases do desenvolvimento, no intuito de identificar a curva que esses dados poderão produzir. Parece que o desenvolvimento de estudos que comparem desde crianças pré-escolares até jovens adultos pode auxiliar na identificação dos mecanismos de distorção mnemônica e no entendimento do próprio processo memória/esquecimento.

Outras variáveis também devem ser consideradas no estudo da memória de crianças, como a forma de apresentação do material, a carga emocional do material utilizado e o momento da testagem. Khanna e Cortese (2009) estudaram a memória de crianças para palavras apresentadas visualmente e palavras apresentadas oralmente e constataram que as crianças tiveram um melhor desempenho nestas últimas. Com relação à emocionalidade do material, Howe (2007) sugere que crianças de oito anos apresentaram mais falsas memórias para as listas de palavras semanticamente associadas com conteúdo neutro do que com conteúdo negativo. Já Carneiro, Albuquerque, Fernandez, e Esteves (2007) sugerem que o intervalo entre a fase de estudo e a fase de teste também parece estar associado às falsas memórias em crianças, sendo um maior intervalo responsável pelo aumento das falsas memórias. Todos estes estudos, não obstante, foram realizados apenas com crianças em idade escolar, sendo pouco o que se sabe sobre o funciomanento da memória na idade pré-escolar.

\section{REFERÊNCIAS}

Barbosa, M. E., Ávila, L. M., Feix, L. F., \& Grassi-Oliveira, R. (2010). Falsas memórias e diferenças individuais. In L. M. Stein e cols. (Org.), Falsas memórias: fundamentos científicos e suas aplicações clínicas e jurídicas (pp. 133-154). Porto Alegre: Artmed.

Brainerd, C. J., \& Mojardin, A. H. (1998). Children's and adult's spontaneous false memories: long-term persistence and meretesting effects. Child Development, 69(5), 1361-1377.

Brainerd, C. J., \& Reyna, V. F. (1998). Fuzzy-trace theory and children's false memories. Journal of Experimental Child Psychology, 71(2), 81-129.

Brainerd, C. J., \& Reyna, V. F. (2005). The science of false memory. New York: Oxford University.

Brainerd, C. J., \& Reyna, V. F. (2007). Explaining developmental reversals in false memory. Psychological Science, 18(5), 442-448.
Brainerd, C. J., Forrest, T. J., Karibian, D., \& Reyna, V. F. (2006). Development of the false-memory illusion. Developmental Psychology, 42(5), 962-979.

Brainerd, C. J., Reyna, V. F., \& Ceci, S. J. (2008). Developmental reversals in false memory: a review of data and theory. Psychological Bulletin, 134(3), 343-382.

Brainerd, C. J., Reyna, V. F., \& Forrest, T. J. (2002). Are young children susceptible to the false-memory illusion? Child Development, 73(5), 1363-1377.

Bruck, M., Ceci, S. J., \& Hembrooke, H. (2002). The nature of children's true and false narratives. Developmental Review, 22, 520-554.

Carneiro, P., Albuquerque, P., Fernandez, A., \& Esteves, F. (2007). Analyzing false memories in children with associative lists specific for their age. Child Development, 78(4), 1171-1185.

Henry, L. A., \& Gudjonsson, G. H. (2004). The effects of memory trace strength on eyewitness recall in children with and without intellectual disabilities. Journal of Experimental Child Psychology, 89, 53-71.

Howe, M. L. (2005). Children (but not adults) can inhibit false memories. Psychological Science, 16(12), 927-931.

Howe, M. L. (2007). Children's emotional false memories. Psychological Science, 18(10), 856-860.

Khanna, M. M., \& Cortese, M. J. (2009). Children and adults are differentially affected by presentation modality in the DRM paradigm. Applied Cognitive Psychology, 23, 859-877.

Lampinen, J. M., Leding, J. K., Reed, K. B., \& Odegard, T. N. (2006). Global gist extraction in children and adults. Memory, 14(8), 952964.

Murdock, B. B., Jr. (1962). The serial position effect on free recall. Journal of Experimental Psychology, 64(5), 482-488.

Neufeld, C. B., \& Stein, L. M. (2003). Falsas memórias em préescolares: uma investigação experimental e suas implicações clínicas. In M. Z. S. Brandão, F. C. S. Conte, F. S. Brandão, Y. K. Ingermann, C. B. Moura, V. M. Silva, \& S. M. Oliane (Orgs.), Sobre comportamento e cognição: clínica, pesquisa e aplicação (Vol. 12, pp. 453-468). Santo André, SP: ESETec.

Neufeld, C. B., Brust, P. G., \& Stein, L. M. (2010). Compreendendo o fenômeno das falsas memórias. In L. M. Stein et al. (Orgs.), Falsas memórias: fundamentos científicos e suas aplicações clínicas e jurídicas (pp. 21-41). Porto Alegre: Artmed.

Pisa, O., \& Stein, L. M. (2006). Entrevista forense de crianças: técnicas de inquirição e qualidade do testemunho. Revista da Ajuris, 23, 217-255.

Powell, M. B., Jones, C. H., \& Campbell, C. (2003). A comparison of preschoolers' recall of experienced versus non-experienced events across multiple interviews. Applied Cognitive Psychology, 17(8), 935-952.

Reyna, V. F., \& Brainerd, C. J. (1995). Fuzzy trace theory: an interim synthesis. Learning and Individual Differences, 7(1), 1-75.

Seamon, J. G., Luo, C. R., Schlegel, S. E., Greene, S. E., \& Goldenberg, A. B. (2000). False memory for categorized pictures and words: the category associates procedure for studying memory errors in children and adults. Journal of Memory \& Language, 42, 120-146.

Stein, L. M. (1998). Memory falsification in children: a developmental study of spontaneous and implanted false 
memories. Tese de doutorado não publicada, University of Arizona, Tucson, EUA.

Sugrue, K., \& Hayne, H. (2006). False memories produced by children and adults in the DRM Paradigm. Applied Cognitive Psychology, 20, 625-631.

Welter, C. L. W., \& Feix, L. F. (2010). Falsas memórias, sugestionabilidade e testemunho infantil. In L. M. Stein et al. (Orgs.). Falsas memórias: fundamentos científicos e suas aplicações clínicas e jurídicas (pp. 157-185). Porto Alegre: Artmed.

Endereço para correspondência:

Carmem Beatriz Neufeld. Universidade de São Paulo - USP. Av. Bandeirantes, 3.900, CEP 14040-901, Ribeirão Preto-SP, Brasil.E-mail: cbneufeld@ffclrp.usp.br. 